\title{
Recomendaciones para el tamizaje del cáncer cervicouterino en mujeres mayores de 65 años. Revisión monográfica
}

\section{Recommendations for cervico-uterine cancer screening in women older than 65 years. Monographic review}

\author{
Antonio Gutiérrez-Ramírez, René Mandujano-Ocampo* y Juan Jiménez-Huerta \\ Departamento de Ginecología y Obstetricia, Hospital Juárez de México, Secretaría de Salud, Ciudad de México, México
}

\begin{abstract}
Resumen
Las recomendaciones de tamizaje para la detección y diagnóstico de lesiones escamosas intraepiteliales de bajo riesgo, alto riesgo y adenocarcinoma invasor, así como su prevención, se basan en el cribado de la citología y de la prueba del virus del papiloma humano. En el presente estudio se obtuvieron resultados similares a los hallazgos internacionales, concluyendo que esta forma de tamizaje es de gran utilidad para su empleo en México. Los Servicios Preventivos Americanos señalan que la pesquisa debe descontinuarse a los 65 años, aunque para la Sociedad Americana de Cáncer la edad de descontinuación debe ser a los 70 años, si hay tres o más citologías negativas y ninguna prueba anormal en los 10 años anteriores.
\end{abstract}

Palabras clave: Citología. Lesiones intraepiteliales de bajo grado. Lesiones intraepiteliales de alto grado. Virus del papiloma humano. Adenocarcinoma. CaCu.

\begin{abstract}
Screening recommendations for the detection and diagnosis of squamous intraepithelial lesions of low risk, high risk and invasive adenocarcinoma and its prevention are based on cytology screening and testing of human papillomavirus. In this study, similar results to international findings were obtained. We concluded that this form of screening is useful for use in Mexico. The American Preventive services indicates that the investigation should be discontinued at 65 years of age, although for the American Cancer Society the age of discontinuations should be at age 70, if there are 3 or more negative Pap smears and no abnormal test in the previous 10 years.
\end{abstract}

Key words: Cytology. Low-grade intraepithelial lesions. High-grade intraepithelial lesions. Human papillomavirus. Adenocarcinoma. CC.

\section{Introducción}

El cáncer del cérvix ( $\mathrm{CaCu}$ ) es un problema de salud para las mujeres de todo el mundo, en el que constituye el segundo tipo de cáncer. Cada año ocurren aproximadamente 400,000 nuevos casos, de los cuales el $80 \%$ son en países en vías de desarrollo. Anualmente mueren por lo menos un cuarto de millón de mujeres. América Latina es la segunda región más afectada por este problema de salud ${ }^{1}$.
Correspondencia:

*René Mandujano-Ocampo

E-mail: mandujanoor@ hotmail.com
Disponible en internet: 13-11-2020 Rev Hosp Jua Mex. 2020;87(3):140-144

www.revistahospitaljuarez.com 1405-9622/○ 2020 Sociedad Médico-Quirúrgica del Hospital Juárez de México, A.C. Publicado por Permanyer. Este es un artículo open access bajo la licencia CC BY-NC-ND (http://creativecommons.org/licenses/by-nc-nd/4.0/). 
El CaCu ha ocupado en México el primer lugar entre los tumores malignos en la población femenina, con una tasa de mortalidad de 18.3 por 100,000 mujeres de 25 y más años. El $24.4 \%$ del total de casos nuevos de neoplasias malignas corresponden a $\mathrm{CaCu}$. De esta cifra, el $47 \%$ de los casos se presentaron en mujeres de 35 a 54 años de edad ${ }^{1,2}$.

En los países desarrollados, la implementación de programas basados en la citología ha logrado reducir significativamente la incidencia y mortalidad por $\mathrm{CaCu}$. Sin embargo en países latinoamericanos se han topado con algunas limitaciones, de las cuales las principales son: baja cobertura de mujeres tamizadas, bajo porcentaje de efectivamente seguidas y tratadas, y sensibilidad de la prueba entre moderada y baja $a^{3,4}$.

El cribado tiene como objetivo detectar lesiones precursoras en el epitelio cervical que serían el antecedente del cáncer invasor. Sin embargo, por varias razones, en ningún programa se ha logrado erradicar totalmente la enfermedad ${ }^{5,6}$.

La incidencia de $\mathrm{CaCu}$ invasor está relacionado con la edad, siendo la media de 47 años en EE.UU.

Se ha señalado que las pacientes con carcinoma in situ no tratadas progresan a carcinoma invasor en un $30 \%$ en los primeros 10 años de seguimiento y en alrededor del $80 \%$ en los 30 años después del diagnóstico 7,8 .

\section{Factores de riesgo}

Un factor de riesgo es aquel que aumenta las probabilidades de que una población padezca una enfermedad. A partir de la década de 1980 se ha identificado al virus del papiloma humano (VPH) como una causa para desarrollar la enfermedad. Hay informes de que el $\mathrm{CaCu}$ invasor se asocia con la presencia de VPH en el $99.7 \%$ de los casos. Y se ha notificado la existencia de más de 100 tipos de VPH, siendo las variantes 16, 18, $31,33,35,39,45,51,52,56,58,59$ y 68 las de más alto riesgo oncogénico, por encontrarse asociadas al CaCu en más del $95 \%$ de los casos. La mayoría de las infecciones por VPH son subclínicas, detectables por la citología o la colposcopia, o bien latentes, detectables únicamente por análisis de ADN viral. Solo el 1\% de los adultos sexualmente activos presenta lesiones clínicas en forma de condilomas. En el $75 \%$ de los carcinomas in situ y el $100 \%$ de los carcinomas infiltrantes del cérvix se hallan ciertos tipos de VPH considerados de riesgo; de ellos, el $75 \%$ corresponde a los serotipos 16 (el más frecuente) o 18 (el más oncogénico) ${ }^{7,9,10}$.
El tabaquismo es otro factor, ya que las fumadoras tienen el doble de probabilidades respecto a las no fumadoras de padecer $\mathrm{CaCu}$.

El virus de inmunodeficiencia humana (VIH) ocasiona daño al sistema inmunológico y provoca que las mujeres estén en mayor riesgo de infección por VPH.

Otro grupo de mujeres en riesgo del $\mathrm{CaCu}$ son aquellas que reciben medicamentos para suprimir sus respuestas inmunitarias en casos como enfermedad autoinmune o de un trasplante de órgano.

Otros factores de riesgo son: nivel socioeconómico bajo, inicio temprano de la vida sexual, antecedente de dos o más parejas sexuales, la edad temprana del primer embarazo, tres o más partos y el uso de anticonceptivos hormonales ${ }^{5}$.

\section{Historia natural}

Tanto la mujer como el hombre pueden ser portadores asintomáticos y vehículos de la infección genital por VPH. La transmisión se produce por contactos sexuales y los órganos más susceptibles de infección con potencial de iniciar una transformación neoplásica son el cuello uterino (zona de transición) y la línea pectina del canal anal. Una de las razones por la que este tipo de infecciones ha cobrado un gran interés reside en la asociación etiológica de algunas de estas infecciones con el $\mathrm{CaCu}$ y con otros tumores del tracto anogenital masculino y femenino.

La prevalencia de VPH está asociada a la edad, siendo más alta en las edades inmediatas al inicio de las relaciones sexuales. En poblaciones donde el número de compañeros sexuales distintos y ocasionales es elevado, la prevalencia puede ser del 30 al $40 \%$ en los grupos de 15 a 25 años de edad. A esto sigue una disminución muy marcada en las edades intermedias (25 a 40 años). Las características de la historia natural de la infección por VPH están también relacionadas con el tipo viral relacionado. El grupo de VPH asociados a alto riesgo neoplásico (unos 15 tipos virales) tiende a establecer infecciones persistentes y a progresar con mayor frecuencia que el grupo de tipos de bajo riesgo. En un estudio de grandes dimensiones realizado en Guanacastle (Costa Rica) las infecciones por VPH de tipo 16 y 18 progresaron a lesiones de neoplasia intraepitelial cervical (CIN) III, en un 17.2 y un $13.6 \%$ respectivamente, mientras que las infecciones por otros tipos virales de alto riesgo progresaron a un $3.0 \%$. Las diferencias son estadísticamente significativas ${ }^{9,10}$. 
Tabla 1. Cribado con citología y test del ADN de VPH-AR (a partir de 35 años). Conducta según resultados

\begin{tabular}{|c|c|c|c|}
\hline Citología & $\begin{array}{l}\text { ADN- } \\
\text { VPH-AR }\end{array}$ & Conducta 1 & Conducta 2 \\
\hline \multirow[t]{2}{*}{ Negativa } & Negativo & $\begin{array}{l}\text { Cribado a los } 5 \\
\text { años }\end{array}$ & \\
\hline & Positivo & $\begin{array}{l}\text { Citología y } \\
\text { ADN-VPH a } \\
\text { los } 12 \text { meses }\end{array}$ & $\begin{array}{l}\text { Ambas negativas: } \\
\text { cribado Alguna } \geq \\
\text { ASC-US: colposcopia }\end{array}$ \\
\hline \multirow[t]{2}{*}{ ASC-US } & Negativo & $\begin{array}{l}\text { Citología al } \\
\text { año }\end{array}$ & $\begin{array}{l}\text { Si negativa: cribado a } \\
5 \text { años }\end{array}$ \\
\hline & Positivo & Colposcopia & \\
\hline $\begin{array}{l}\text { LSIL*HSIL, } \\
\text { ASC-H } \\
\text { AGC-AIS }\end{array}$ & $\begin{array}{l}\text { Negativo } \\
0 \\
\text { Positivo }\end{array}$ & Colposcopia & \\
\hline $\begin{array}{l}\text { *Alternativa } \\
\text { LSIL }\end{array}$ & Negativo & $\begin{array}{l}\text { Citología a los } \\
6 \text { y } 12 \text { meses }\end{array}$ & $\begin{array}{l}\text { Ambas negativas: } \\
\text { cribado Alguna } \geq \\
\text { ASC-US: Colposcopia }\end{array}$ \\
\hline
\end{tabular}

VPH-AR: Cepas del virus del papiloma humano de alto riesgo; ASC-US: Atypical Squamous Cells of Indetermined Significance; LSIL: Low Squamous Intraepitelial Leson; HSIL: High Squamous Intraepitelial Leson; ASC-H: Atypical Squamous Cells High; AGC-AIS: Atypical Glandular Cells- Atypical Intraepitelial Squamous.

El mecanismo conocido como inducción neoplásica por VPH se produce a partir de la síntesis de las proteínas virales E6 y E7. Estas proteínas se ligan a las proteínas producidas por los genes supresores de tumores $P 53$ y $R B$ respectivamente, agrandándolas e inutilizándolas funcionalmente. Esta interacción en células proliferativas, como las del cuello uterino, y especialmente de la zona de unión escamocilíndrica con un epitelio inestable, impide la correcta reparación del ADN, conduce a una inestabilidad genómica y aumenta la probabilidad de desarrollar mutaciones específicas, esenciales para la progresión a cáncer invasor (Tabla 1).

\section{Estrategias de tamizaje}

Se recomienda que el programa de pesquisa se inicie tres años después del inicio de vida sexual, pero nunca más tarde de los 21 años. También se señala que la pesquisa debe descontinuarse a los 65 años $^{11}$. Reportes previos han señalado que cuando una citología reporta una lesión escamosa intraepiteal de alto grado (o HSIL, por sus siglas en inglés), el riesgo de enfermedad cervical es elevado. Un examen colposcópico en esos casos identifica CIN II o más en el 53 al $66 \%$ de los casos, y cuando se realiza una escisión electroquirúrgica se puede detectar CIN II en por lo menos el 84 al $97 \%$ de los casos $^{12}$. Aproximadamente un $2 \%$ de las pacientes con citología positiva de lesión escamosa intraepitelial (LEI) de alto grado tiene cáncer invasor. Los hallazgos de este estudio se relacionan bien con estos reportes. Al hacer la colposcopia predominó el hallazgo. Esta incidencia se ha mantenido a lo largo de las últimas dos décadas ${ }^{13}$.

La incidencia pico para carcinoma in situ del cérvix es alrededor de 30 a 35 años, mientras que para el invasivo es cerca de los 50 . Por esta razón los programas de tamizaje concentran su atención en el grupo de 30 a 50 años.

Se recomienda que se prioricen también las mujeres de mayor edad que nunca se hayan hecho la prueba de Papanicolaou, ya que más del $25 \%$ de los casos de $\mathrm{CaCu}$ invasivo ocurren en mujeres mayores de 65 años; y del 40 al $50 \%$ de mujeres que mueren de $\mathrm{CaCu}$ tienen más de 65 años ${ }^{14}$.

Se ha comprobado que la causa necesaria del $\mathrm{CaCu}$ es la infección por el VPH, cuya principal vía de transmisión es la sexual ${ }^{7}$. Existe una asociación de más del 99\% entre el VPH y el CaCu ${ }^{8,9}$. La prueba de VPH por captura híbrida es una tecnología de biología molecular que detecta la presencia de ADN de los 13 tipos de VPH considerados de alto riesgo oncogénico en las células del cuello del útero, por medio de un sistema de captura de híbridos (ensayo de hibridación de ácido nucleico). Estos 13 tipos detectados son de utilidad para el tamizaje de cáncer cervical y para muchos aspectos del manejo clínico de la prevención ${ }^{15}$.

La prueba de VPH es significativamente más sensible que la citología para detectar CIN II y tiene un alto valor predictivo negativo cercano al $100 \%^{16}$. Un estudio realizado en Italia demostró que la prueba de VPH es más efectiva que la citología para detectar CIN II en una primera ronda de tamizaje ${ }^{17}$. Esta evidencia sustenta la recomendación de ampliar el intervalo tamizaje con seguridad entre tres y cinco años. En México, el programa nacional de detección de $\mathrm{CaCu}$ establece un intervalo de frecuencia para la prueba de VPH negativa de cinco años ${ }^{18}$.

En mujeres con citología anormal y prueba de VPH positiva, la recomendación de la Organización Mundial de la Salud es la derivación a colposcopia y biopsia, y tratamiento posterior a la confirmación histológica ${ }^{19}$.

Las mujeres mayores de 65 años, con pruebas negativas de supervisión previa, y sin antecedentes de CIN II dentro de los últimos 20 años, no deberían hacerse la prueba de detección de $\mathrm{CaCu}$ en cualquier modalidad. Y una vez que el cribado es interrumpido, no se debe reanudar por cualquier motivo, incluso si la mujer informa que tiene una nueva pareja sexual ${ }^{20}$. 


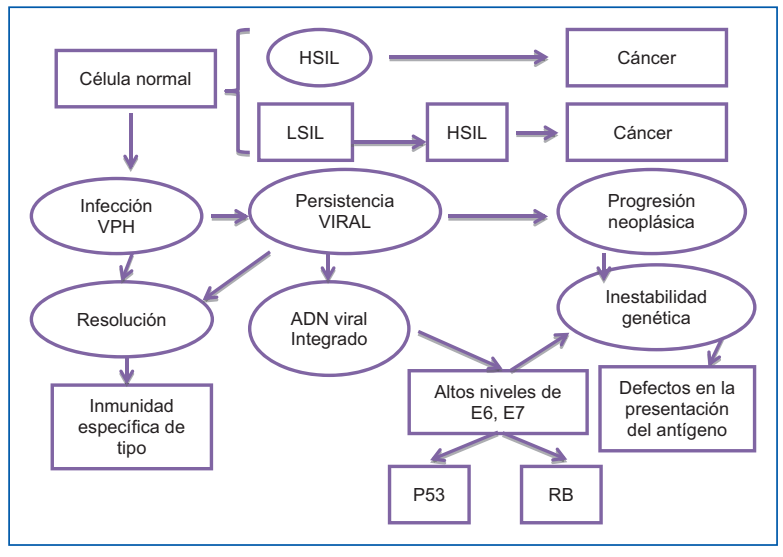

Figura 1. Mecanismo de inducción neoplásica por virus de papiloma humano de alto riesgo. LSIL: Low Squamous Intraepitelial Leson; HSIL: High Squamous Intraepitelial Leson; VPH: virus del papiloma humano.

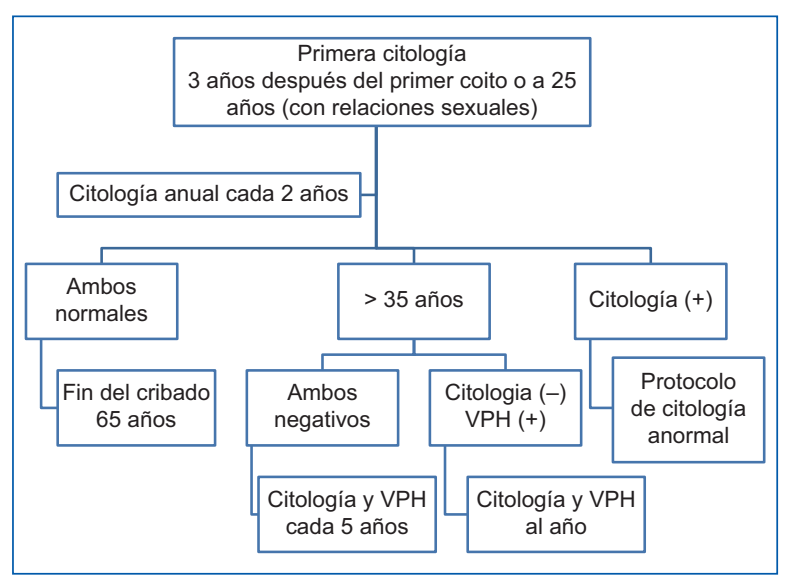

Figura 2. Cribado de cáncer cervical.

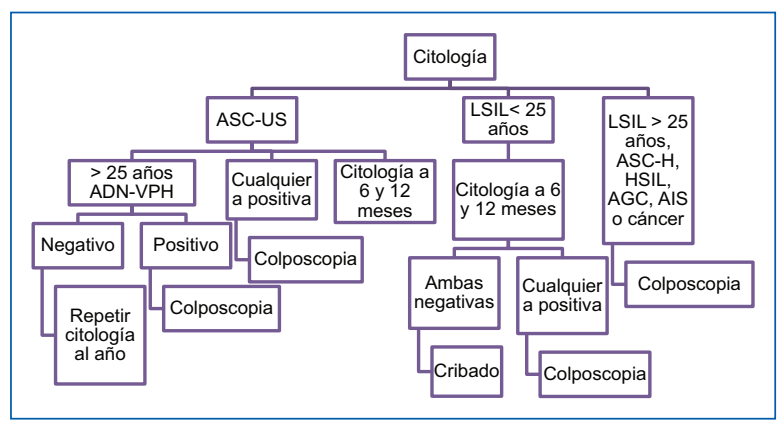

Figura 3. Citología anormal, conducta. ASC-US: Atypical Squamous Cells of Indetermined Significance; LSIL: Low Squamous Intraepitelial Leson; ASC-H: Atypical Squamous Cells High; HSIL: High Squamous Intraepitelial Leson; AGC: Atypical Glandular Cells; AIS: Atypical Intraepitelial Squamous; VPH: virus del papiloma humano.

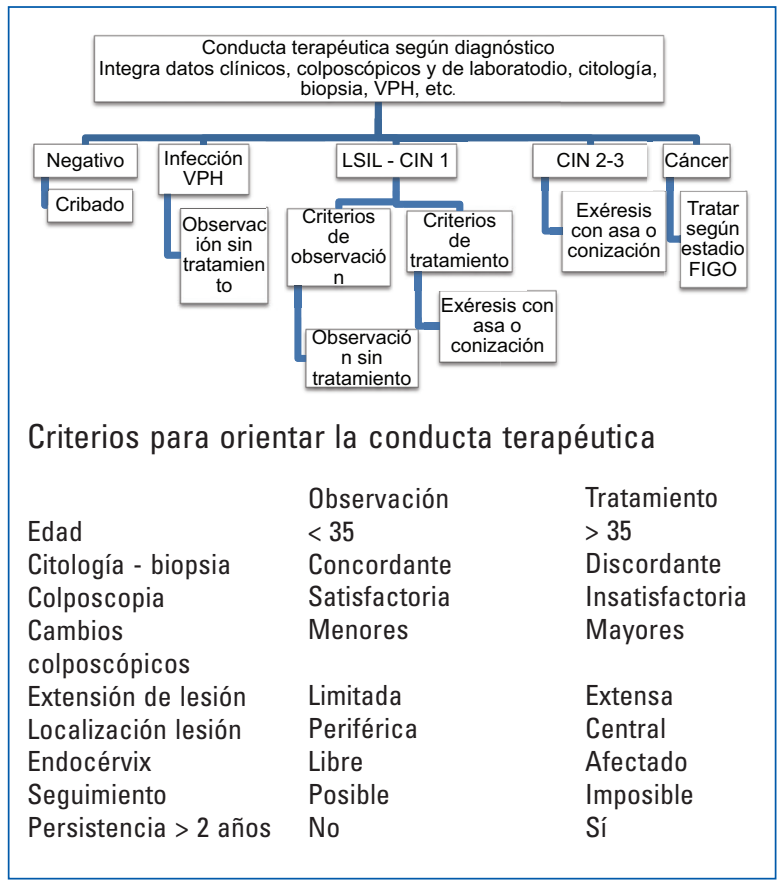

Figura 4. Algoritmos de conducta terapéutica. VPH: virus del papiloma humano; LSIL: Low Squamous Intraepitelial Leson; CIN: neoplasia intraepitelial cervical; FIGO: Federación Internacional de Ginecología y Obstetricia.

En las mujeres con edades mayores a los 65 años con antecedente de CIN II, CIN III o adenocarcinoma in situ, la periodicidad del screening debe continuar durante al menos 20 años más (Figs. 1-4).

\section{Conclusiones}

El CaCu es la segunda causa mundial de muerte por cáncer en mujeres entre los 35 y 64 años. Las principales limitantes son: el bajo porcentaje con resultados en la prueba de Papanicolaou anormal que son efectivamente seguidas y tratadas, y la sensibilidad de la prueba, entre moderada y baja.

La incidencia pico para el carcinoma in situ del cérvix es alrededor de 30 a 35 años, mientras que para el invasivo es cerca de los 50 . Del 40 al $50 \%$ de las mujeres que mueren de $\mathrm{CaCu}$ tiene más de 65 años. Los servicios preventivos americanos recomiendan que el programa de pesquisa se inicie tres años después del inicio de la actividad sexual, pero nunca más tarde de los 21 años, y que la pesquisa debe descontinuarse a los 65 años si hay tres o más citologías negativas y ninguna prueba anormal en los 10 años anteriores. La importancia del diagnóstico temprano se debe no solo 
a la detección de lesiones preinvasivas, sino también a la enfermedad invasiva. La supervivencia de un paciente con cáncer invasivo del cérvix depende del estadio en el momento del diagnóstico. Esta es del $88 \%$ a los cinco años para mujeres con enfermedad localizada al momento del diagnóstico, pero se reduce a solo al 13\% para aquellas con enfermedad distante.

\section{Financiamiento}

La presente investigación no ha recibido ayudas específicas provenientes de agencias del sector público, sector comercial o entidades sin ánimo de lucro.

\section{Conflicto de intereses}

Los autores declaran no tener conflicto de intereses alguno.

\section{Responsabilidades éticas}

Protección de personas y animales. Los autores declaran que para esta investigación no se han realizado experimentos en seres humanos ni en animales.

Confidencialidad de los datos. Los autores declaran que han seguido los protocolos de su centro de trabajo sobre la publicación de datos de pacientes.

Derecho a la privacidad y consentimiento informado. Los autores declaran que en este artículo no aparecen datos de pacientes.

\section{Bibliografía}

1. Ferlay J, Bray F, Pisani P, Parkin DM. GLOBOCAN 2002: CANCER incidence, mortality and prevalence worldwide. Lyon: IARC; 2004.

2. Lewis MJ. Análisis de la situación del cáncer cérvico-uterino en América Latina y el Caribe [Internet]. Washington, DC: Organización Panamericana de la Salud, Organización Mundial de la Salud; 2004. Disponible en: https://iris.paho.org/handle/10665.2/762

3. American Cancer Society. Cancer facts \& figures 2014. Atlanta: American Cancer Society; 2014.
4. ACOG committee opinion. New PaP test screening techniques. Number 206, agust 1998. Committee on Gynecologic Practice. American College of Obstetricians and Gynecologists. Int J Gynaecol Obstet. 1998; 63:312-4.

5. Guidelines for the prevention and Early Detection of cervical Cancer. (published online ahead of print March 14, 2012) CA Cancer J Clin. 2012;62(3). Doi: 10.3322/caac.21139.

6. ACOG Committee on Practice Bulletins-Gynecology. ACOG Practice Bulletin no. 109: cervical cytology screening. Obstet Gynecol. 2009;114:1409-20.

7. Walboomers JM, Jacobs MV, Manos MM, Bosch FX, Kummer JA, Shah KV, et al. Human papillomavirus is a necessary cause of invasive cervical cancer worldwide. J Pathol. 1999:189:12-9.

8. Castellsagué X, Díaz M, de Sanjosé S, Muñoz N, Herrero R, Franceschi S, et al. The worldwide human papillomavirus etiology of cervical adenocarcinoma and its cofactors: implications for screening and prevention. $J$ Natl Cancer Inst. 2006;98(5):303-15.

9. Khan MJ, Castle PE, Lorinez AT, Wacholder S, Sherman M, Scott DR, et al. The elevated 10-year risk of cervical precancer and cancer in women with human papillomavirus (HPV) type 16 or 18 and the possible utility of type-specific HPV testing in clinical practice. J Natl Cancer Inst. 2005;97(14):1072-9.

10. De Sanjosé S, Díaz M, Castellsagué X, Clifford G, Muñoz N, Bosch FX. Worldwide prevalence and genotype distribution of cervical HPV DNA in 169-341 women with normal cytology. A metal-analysis. Lancet Infectious Diseases. 2007:01(7):453-9.

11. Bosch FX, Lorincz A, Muñoz N, Meijer CJ, Shah KV. The causal relation between human papillomavirus and cervical cancer. J Clin Pathol. 2002:55(4):244-65.

12. Sawaya GF, Mc Connell KJ, Kulasingam SL, Lawson HW, Kerlikowske K, Melnikow J, et al. Risk of cervical cancer associated with extending the interval between cervical-cancer screenings. N Engl J Med. 2003; 349:1501-9.

13. Zapka J, Tapplin S, Anhang Price R, Cranos C, Yabroff R. Factors in quality care - The case of follow-up to abnormal cancer screening test - Problems in the steps and interfaces of care. J Natl Cancer Inst Monogr. 2010;40:58-71

14. Sasieni $P$, Castanon A, Cuzick J. Effectiveness of cervical screening with age: population based case-control study of prospectively recorded data. BMJ. 2009,339:b2968.

15. Wright TC Jr, Schiffman M. Adding a test for human papillomavirus DNA to cervical-cancer screening. N Engl Med. 2003;348:489-90.

16. Rijkaart DC, Berkhof J, Rozendaal L, van Kemenade FJ, Bulkmans NWJ, Heideman DAM, et al. Human papillomavirus testing for the detection of high-grade cervical intraepithelial neoplasia and cancer: final results of the POBASCAM randomised controlled trial. Lancet Oncol. 2012; 13:78-88.

17. Dillner J, Rebolj M, Birembaut P, Petry KU, Szarewski A, Munk C, et al. Long term predictive values of cytology and human papillomavirus testing in cervical cancer screening: joint European cohort study. BMJ. 2008;337:a1754.

18. Bulkman NW, Berkhof J, Rozendaal L, van Kemenade FJ, Boeke AJP Bulk $S$, et al. Human papillomavirus DNA testing for the detection of cervical intraepithelial neoplasia grade 3 and cancer: 5 year follow-up of a randomized controlled implementation trial. Lancet. 2007;370: 1764-72.

19. Wright T, Cox J, Massad L, Twiggs L, Wilkinson E. ASCCP-Sponsored Consensus Conference. 2001 Consensus Guidelines for the management of women with cervical cytological abnormalities. JAMA. 2002;287:2120-9.

20. ASCUS-LSIL Traige Study (ALTS) Group. A randomized trial on the management of low-grade squamous intraepithelial lesion cytology interpretations. Am J Obst Gin. 2003;188:1393-400. 